\title{
The Distributions of the Density and the Mass for Spiral Galaxies
}

\author{
Nazzeha A. Daod \\ Department of Physics \\ College of Science \\ University of Mosul
}

(Received 14/8/2011; Accepted 21/12/2011)

\begin{abstract}
Expressions for the distributions of the density and mass of spiral galaxies have been derived for three different orientations of rotation curves with a turn over radius $\left(\mathrm{r}_{\mathrm{t}}\right)$. The central region of the galaxy is found to rotate as a rigid body with a constant density and with a central mass as a function of $r^{3}$. The density of the outer regions $\left(r>r_{t}\right)$ is found to vary inversely with the radius (r), and the corresponding mass is found to be a function of $r^{2}$. Galaxies with flat rotation curves at $\left(r>r_{t}\right)$ has less mass than that with rising up or lowering down rotation curves.
\end{abstract}

Keywords: Rotation curve, density law, spiral galaxies.

\section{توزبعات الكنلفة والكنلة المجرل الحازونية}

\section{الملخص}

في هذا البحث مُ لشققاق علاقات لكتلفة وكتلة المجرات الحلزونية لثلاثة نماذج مختلفة لمنحنيت

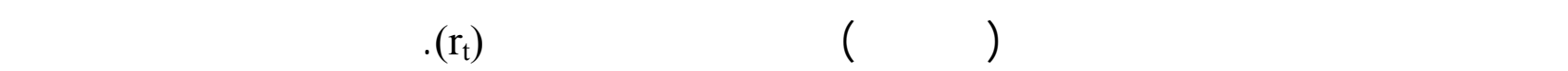

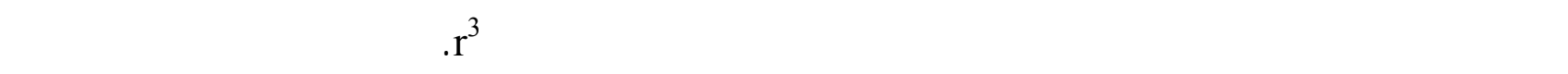

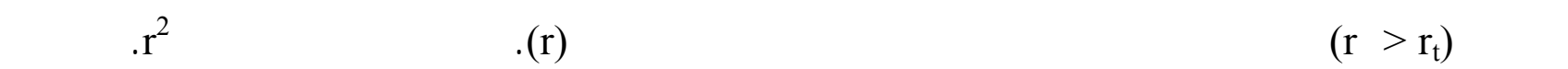
بأن المجرات التي تمنلك منحنيلت منبطة عند (r > r r تمتلك أقل كثلفة من المجرات التي ترقفع منحنياتها إلى الأعلى أو التي تنحدر منحنياتتها إلى الأسفل قليليلاً. الكاملت الدالة: المنحنيت الدورانية، قانون الكثافة، المجرات اللولبية (الحلزونية).

\section{INTRODUCTION}

The rotation of spiral galaxies was discovered by Slipher (1914) when he detected inclined absorption lines in the spectrum of M31 galaxy.

Rotation curves of spiral galaxies are one of the main tools to study the distribution of mass in spiral galaxies. They constitute the best observational proof for the existence of dark matter in spiral galaxies. Rotation curves provide important information for understanding the dynamics, evolution and formation of galaxies. 
Rotation curves which are useful to derive the mass distribution in spiral galaxies are obtained by observing the emission lines of ionized gas at optical frequencies such as $\mathrm{H} \alpha$ or the $21 \mathrm{~cm}$ line of neutral hydrogen at radio frequencies (wright, 1971; Roberts and Rots, 1973).

The main tool that we had used for this study is the rotation curves of spiral galaxies. Although its already 50 years that the rotation curves are used in order to derive the matter distribution (density law of galaxies and galactic mass) in spirals, a different approaches can be always applied to get a more relevant information. For this purpose we started with what is called a density law for spiral galaxies derived by Daoud et al., 2009, which is a solution of a general Burbidge equation related the galactic velocity to the galactic density (Binney and Tremaine, 1987).

In this paper we studied the galactic density and mass of spirals that their rotation curves show a turn over at some distance from the center of the galaxy (radius) $r_{t}$, like NGC 4378, NGC 4594, NGC 3145, NGC 7664 and others Fig. (1).



Fig. 1: Series of rotation curves for spiral galaxies (Figure from Rubin, Ford and Thonnard, AP. J. Lett. 255. L107, 1978. Cited in Mihalas and Binney, 1968).

At radii less than the turn over radius $\left(r<r_{t}\right)$ it has been found that the behavior of the density and the mass for all galaxies are the same. i.e. the central density is found to be a constant while the central mass turns to be a function of $\mathrm{r}^{3}$.

At radii larger than the turn over radius $\left(r<r_{t}\right)$, the rotation curves of spiral galaxies show three different cases. In case one the curve goes up slightly as in NGC 4594, in case two the curve is almost flat as in NGC 3145), while in case three, the curve goes down slightly (NGC 4378). In all the three cases mentioned above the galactic density at $\left(r>r_{t}\right)$ is inversely proportional to the radius ( $\left.r\right)$, therefore, the density decreases for large radii. In case 2 where the curve is flat, we found that the galactic mass for the outer 
regions of the galaxy is very small comparing with the other two cases, while the mass of the outer regions in case 3 is the largest.

\section{CALCULATIONS AND RESULTS}

To study the density of spiral galaxies we started with the following equation:

$$
\rho(r, k)=\frac{k^{4}}{\pi^{2} \mathrm{G} \sqrt{1-k^{2}} \mathrm{r}} \frac{\mathrm{d}}{\mathrm{dr}} \frac{1}{\mathrm{r}} \int_{\mathrm{o}}^{\mathrm{r}} \frac{\mathrm{V}^{2}(\mathrm{a}) \mathrm{ada}}{\sqrt{\mathrm{r}^{2}-\mathrm{k}^{2} \mathrm{a}^{2}}}
$$

from (Daoud et al., 2009), where $\rho$ is the galactic density, $\mathrm{k}$ is the eccentricity of the system and $\mathrm{V}(\mathrm{a})$ is the velocity at distance a from the center of the galaxy.

Rotation curves of spiral galaxies with a turn over radius take different orientations at $\left(r>r_{t}\right)$ as follows.

\section{Case 1 [The curve abc 1 ]:}

Some spiral galaxies show rotation curves $\left(a b c_{1}\right)$ which rise up slightly at $\left(r>r_{t}\right)$ (the curve bc1 in Fig. 2).

In this case rotation curves can be approximated by two linear equations.

$$
\mathrm{V}(\mathrm{a})=\alpha \mathrm{a} \text { at } \mathrm{a}<\mathrm{r}_{\mathrm{t}}
$$

Where $\alpha$ is a constant which refers to the slope of the inner part of the rotation curve (the slope of the line ab Fig. 2).

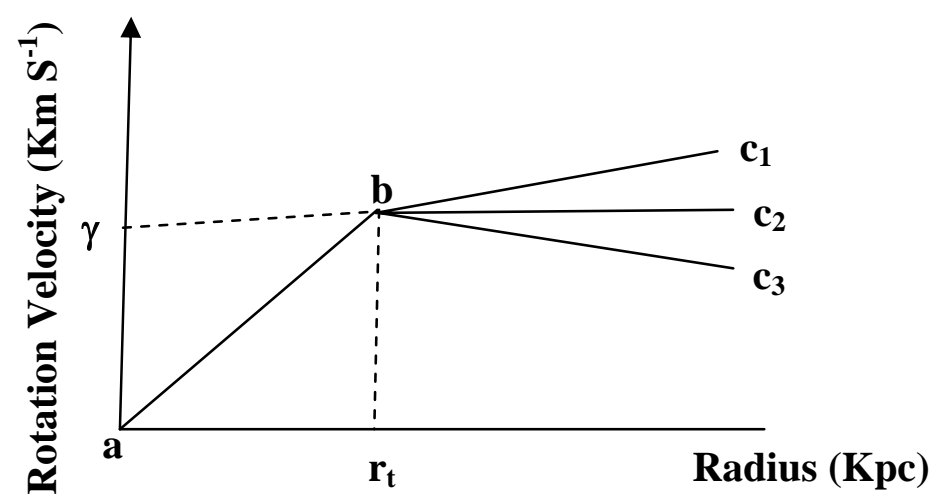

Fig. 2: Three general forms of rotation curves for spiral galaxies

and

$$
\mathrm{V}(\mathrm{a})=\beta \mathrm{a}+\gamma \quad \text { at } \mathrm{a}>\mathrm{r}_{\mathrm{t}}
$$

Where $\beta$ is a constant which refers to the slope of the outer part of the rotation curve (the slope of the line $b_{1}$ in Fig. 2), and $\gamma$ is another constant which can be found from the intersection of the outer part of the rotation curve $\left(b c_{1}\right)$ with the vertical axis.

Let $\rho_{1}(r, k)$ be the density of the inner region of the galaxy $\left(r<r_{t}\right)$ where $V(a)=\alpha a$.

Substituting equation (2) in equation (1) and letting $x=\frac{r}{k}$ we have. 


$$
\rho_{1}(\mathrm{r}, \mathrm{k})=\frac{\mathrm{k}^{3} \alpha^{2}}{\mathrm{G} \pi^{2} \sqrt{1-\mathrm{k}^{2}} \mathrm{r}} \frac{\mathrm{d}}{\mathrm{dr}}\left[\frac{1}{\mathrm{r}} \int_{\mathrm{o}}^{\mathrm{r}} \frac{\mathrm{a}^{3} \mathrm{da}}{\sqrt{\mathrm{x}^{2}-\mathrm{a}^{2}}}\right]
$$

Using standard integral (William and Beyyer, 1985).

$$
\int_{0}^{\infty} \frac{\mathrm{a}^{3} \mathrm{da}}{\sqrt{\mathrm{x}^{2}-\mathrm{a}^{2}}}=-\frac{1}{3} \sqrt{\mathrm{x}^{2}-\mathrm{a}^{2}}\left(\mathrm{a}^{2}+2 \mathrm{x}^{2}\right)
$$

And then differentiating we get.

$$
\rho_{1}(\mathrm{r}, \mathrm{k})=\frac{2 \alpha^{2}\left\{2-\sqrt{1-\mathrm{k}^{2}}\left(\mathrm{k}^{2}+2\right)\right\}}{3 \mathrm{G} \pi^{2} \sqrt{1-\mathrm{k}^{2}}}
$$

Equation (6) shows that the density of the inner parts of the galaxy is in depended of $r$ ( $\rho$ is constant), this means that the central part of the galaxy rotates like a rigid body which is consistence with the texts (Mihalas and Binney, 1968 ; Carroll and Ostlie, 1996).

To find an expression for the mass of the galaxy, we use the following equation.

$$
\mathrm{dm}=4 \pi \mathrm{a}^{2} \sqrt{1-\mathrm{k}^{2}} \rho(\mathrm{a}, \mathrm{k}) \mathrm{da}
$$

Since we divided the rotation curve into two regions then let us first find the inner mass of the galaxy $\mathrm{m}_{1}(\mathrm{r})$, with the central density $\rho_{1}(\mathrm{r}, \mathrm{k})$ at $\left(\mathrm{r}<\mathrm{r}_{\mathrm{t}}\right)$.

$$
\mathrm{m}_{1}(\mathrm{r})=4 \pi \sqrt{1-\mathrm{k}^{2}} \int_{\mathrm{o}}^{\mathrm{r}} \rho_{1}(\mathrm{a}, \mathrm{k}) \mathrm{a}^{2} \mathrm{da}
$$

Substituting for $\rho_{1}(\mathrm{a}, \mathrm{k})$ from equation (6) then we have.

$$
\mathrm{m}_{1}(\mathrm{r})=\frac{8 \alpha^{2}\left\{2-\sqrt{1-\mathrm{k}^{2}}\left(\mathrm{k}^{2}+2\right)\right\}}{9 \mathrm{G} \pi} \mathrm{r}^{3}
$$

Therefore the inner mass of the galaxy at $\left(r<r_{t}\right)$ is proportional to $r^{3}$, in other words the inner mass of the galaxy increases sharply as we go farther from the galactic center.

To find the density at $\left(r>r_{t}\right)$, we substitute equation (3) in equation (1), letting $x=\frac{r}{k}$ and using the following standard integrals (William and Beyyer, 1985).

$$
\begin{aligned}
& \int_{0}^{\mathrm{r}} \frac{\mathrm{a}^{3} \mathrm{da}}{\sqrt{\mathrm{x}^{2}-\mathrm{a}^{2}}}=-\frac{1}{3} \sqrt{\mathrm{x}^{2}-\mathrm{a}^{2}}\left(\mathrm{a}^{2}-2 \mathrm{x}^{2}\right) \\
& \int_{\mathrm{r}}^{\mathrm{r}} \frac{\mathrm{a}^{2} \mathrm{da}}{\sqrt{\mathrm{x}^{2}-\mathrm{a}^{2}}}=-\frac{\mathrm{a}}{2} \sqrt{\mathrm{x}^{2}-\mathrm{a}^{2}}+\frac{\mathrm{x}^{2}}{2} \sin ^{-1} \frac{\mathrm{a}}{|\mathrm{x}|} \\
& \int_{\mathrm{o}}^{\mathrm{r}} \frac{\mathrm{ada}}{\sqrt{\mathrm{x}^{2}-\mathrm{a}^{2}}}=-\sqrt{\mathrm{x}^{2}-\mathrm{a}^{2}}
\end{aligned}
$$

Then differentiating we get.

$$
\rho_{2}(\mathrm{r}, \mathrm{k})=\frac{2 \beta^{2}\left\{2-\sqrt{1-\mathrm{k}^{2}}\left(\mathrm{k}^{2}+2\right)\right\}}{3 \mathrm{G} \pi^{2} \sqrt{1-\mathrm{k}^{2}}}+\frac{\beta \gamma \mathrm{k}\left\{\sin ^{-1} \mathrm{k}-\mathrm{k} \sqrt{1-\mathrm{k}^{2}}\right\}}{\mathrm{G} \pi^{2} \sqrt{1-\mathrm{k}^{2}} \mathrm{r}}
$$

$\rho_{2}(r, k)$ should be the density for the outer region of the galaxy (from $r_{t}$ to $r$ ). According to equation (1) the limits of integration cannot be changed, for this reason, in calculating $\rho_{2}(r, k)$ we integrated from zero (at which $V(r)=\gamma$ ) to $r$ (the edge of the galaxy) and then we found the outer density of the galaxy from. 


$$
\rho_{3}(r, k)=\left|\rho_{2}\left(r_{t}, k\right)-\rho_{1}(r, k)\right| \quad \text { at } \quad r>r_{t}
$$

$$
\rho_{3}(\mathrm{r}, \mathrm{k})=\frac{\beta \gamma \mathrm{k}\left\{\sin ^{-1} \mathrm{k}-\mathrm{k} \sqrt{1-\mathrm{k}^{2}}\right\}}{\mathrm{G} \pi^{2} \sqrt{1-\mathrm{k}^{2}}}\left(\frac{1}{\mathrm{r}_{\mathrm{r}}}-\frac{1}{\mathrm{r}}\right)
$$

Therefore the outer density of the galaxy, $\rho_{3}(r, k)$, is proportional to $r^{-1}$ i.e. the halo density decreases as we go farther from the turn over radius. This result is consistent with the result of Carrol and Ostlie (1996).

The outer mass of the galaxy $\left(\right.$ at $\left.r>r_{t}\right)$ is evaluated by combining equations 7 and 13, then

$$
\mathrm{m}_{2}(\mathrm{r})=\frac{4 \beta \gamma \mathrm{k}\left\{\sin ^{-1} \mathrm{k}-\mathrm{k} \sqrt{1-\mathrm{k}^{2}}\right\}}{3 \mathrm{G} \pi \mathrm{r}_{\mathrm{t}}} \mathrm{r}^{3}-\frac{2 \beta \gamma \mathrm{k}\left\{\sin ^{-1} \mathrm{k}-\mathrm{k} \sqrt{1-\mathrm{k}^{2}}\right\}}{\mathrm{G} \pi} \mathrm{r}^{2}
$$
to $\mathrm{r}^{2}$.

Equation (14) indicate that the mass of the outer regions of the galaxy is proportional

The inner mass of any spiral galaxy of this type of rotation curve can be calculated from equation (9) by letting $r=r_{t}$ and the mass of the outer regions of the galaxy can be calculated from equation (14) by letting $r=r_{g}$ where $r_{g}$ is the radius of the given galaxy, therefore the total mass of the galaxy is given by:

$$
\mathrm{m}_{\text {total }}=\mathrm{m}_{1}\left(\mathrm{r}_{\mathrm{t}}\right)+\mathrm{m}_{2}\left(\mathrm{r}_{\mathrm{g}}\right)
$$

$$
\mathrm{m}_{\text {total }}=\frac{8 \alpha^{2}\left\{2-\sqrt{1-\mathrm{k}^{2}}\left(\mathrm{k}^{2}+2\right)\right\}}{9 \mathrm{G} \pi} \mathrm{r}_{\mathrm{t}}^{3}+\frac{4 \beta \gamma \mathrm{k}\left\{\sin ^{-1} \mathrm{k}-\mathrm{k} \sqrt{1-\mathrm{k}^{2}}\right\}}{\mathrm{G} \pi}\left[\frac{2}{3 \mathrm{r}_{\mathrm{t}}} \mathrm{r}_{\mathrm{g}}^{3}-\mathrm{r}_{\mathrm{g}}^{2}\right]
$$

To find the numerical value of the galactic mass (equation 14), the constants $\alpha, \beta, \gamma$ can be taken from the velocity curve, the eccentricity $\mathrm{k}$ can be taken from observations and $r_{t}$ can be approximated from the velocity curve or it can be found by equating equations (6) and (11) so it turns to be

$$
\mathrm{r}_{\mathrm{t}}=\frac{3 \beta \gamma\left\{\mathrm{k} \sin ^{-1} \mathrm{k}-\mathrm{k}^{2} \sqrt{1-\mathrm{k}^{2}}\right\}}{\left(2 \alpha^{2}+2 \beta^{2}\right)\left\{2-\sqrt{1-\mathrm{k}^{2}}\left(\mathrm{k}^{2}+2\right)\right\}}
$$

\section{Case 2 (The curve $\mathbf{a b c}_{2}$ ):}

For the second case, the rotation curves of some spiral galaxies are almost flat $(\beta \rightarrow 0)$ at $r>r_{t}$ (the curve $b c_{2}$, Fig. 2). In this case we don't have to go through all derivations again, but all we have to do is setting $\beta$ very small $(\beta \rightarrow 0)$ in equation (13) and (14). In equation (13) if $\beta$ is very small then $\rho_{3}(r, k)$ is small too as well as $m_{2}(r)$. In other words when the second part of the rotation curve is almost flat then the mass of the outer parts of the galaxy $\mathrm{m}_{2}(\mathrm{r})$ drops down quickly comparing with the inner mass, therefore, the contribution of the halo mass to the total mass of the galaxy is small comparing with case 1 . 


\section{Case 3 (The curve $a b c_{3}$ ):}

The rotation curves of some spiral galaxies goes down slightly at $r>r_{t}$ (the curve $b_{3}$, Fig. 2). In this case the parameter $\gamma$ (the intersection of the second part of the velocity curve $\left(\mathrm{bc}_{3}\right)$ with the vertical axis) is larger than the previous cases. Using large value for $\gamma$ in equation (13) we get larger density $\rho_{3}(\mathrm{r}, \mathrm{k})$, and it follows that the mass of the outer regions of the galaxy, $\mathrm{m}_{2}(\mathrm{r})$ is larger in this case comparing with the other two cases.

\section{DISCUSSION}

A great deal can be learned about the matter in galaxies by studying rotation curves since galactic rotation depends on the distribution of mass.

Rotation curves of all spiral galaxies show a rapid rise in rotation speed with distance out to a few kilo parsecs from the center $\left(r<r_{t}\right)$. This type of rotation is referred to as a rigid body rotation. In this paper we derived an equation for the density of the inner portion of the galaxy, $\rho_{1}(r, k)$, (equation 6), and it was found to be independent of $r\left(\rho_{1}(r, k)=\right.$ constant). Rigid body rotation near the galactic center implies that the mass must be roughly spherically distributed and the density nearly constant. The derived inner mass of the galaxy $m_{1}(r, k)$, (equation 9), depends on $r^{3}$ which is exactly as expected.

In this study we found that the outer galactic density is proportional to $\mathrm{r}^{-1}\left\{\rho_{3}(\mathrm{r}, \mathrm{k}) \propto \mathrm{r}^{-1}\right\}$ (equation 13), while the galactic density number determined by star count (density of stars in the luminous stellar halo), in the portion of the galaxy beyond the solar galactic radius is proportional to $\mathrm{r}^{-3.5}$ and the galactic density determined from spherically distributed mass is proportional to $\mathrm{r}^{-2}$. Our density dependence is very different from that derived by spherical distribution and from that derived by star count. This discrepancy can be explained as follow. The majority of the mass in the galaxy is in the form of nonluminous (dark) matter. Studying rotation curves of spiral galaxies indicate that it must contain large amount of dark matter. Dark matter is not an insignificant issue, observations of galaxies and clusters of galaxies indicate that 90 to 95 percent of the matter in the universe is dark matter; (seeds, 2007; Miller, 1995).

Although galaxies appear to be separate luminous objects, their dark matter halos may actually merge in intergalactic space, so determination of the total mass of an individual galaxy could not be exact. In other words, the edge of the galaxy (or its radius $r_{g}$ ) is not exactly determined.

The mass of the outer regions of all spiral galaxies $\mathrm{m}_{2}(\mathrm{r})$ is found to be proportional to $r^{2}$. The halo mass of galaxies with flat rotation curve at $r>r_{t}$ is less than the halo mass of the other two cases, while the halo mass of galaxies which show a slightly going down curve at $r>r_{t}$ is the largest.

Equation (17) is derived to calculate the turn over radius $\left(r_{t}\right)$ of the given galaxy depending on other factors that can be taken from the rotation curve and from observations.

\section{REFERENCES}

Binney, J.; Termaine, S. (1987). "Galactic Dynamic". Princeton NJ: Princeton Univ., Press. Carroll, B. W.; Ostlie D.A. (1996). "An Introduction to Modern Astrophysics. AddisonWestey Publishing Company. 956 p. 
Daoud, N. A.; Abdul-Kahader, A. J.; Zeki, M. K. (2009). Density Law of Spiral Galaxy Relative to Rotation Curves. J. Raf. Sci., 20(3), 90-96.

Mihalas, D.; Binney, J. (1968). "Galactic Astronomy, Structure and Kinematics". W.H. Freeman an Company. $466 \mathrm{p}$.

Miller, C. (1995). hHP://www.eclipse. net/ cmmiller/

Roberts, M.S.; Rots, A. H. (1973). Comparison of Rotation Curves of Different Galaxy Types. Astron. and Astrophys. 26, 483.

Rubin, F. ; Thonnard (1978). AP. J. Lett. 255, L 107, Cited in Mihalas and Binney (1968).

Seeds, M.A. (2007). "The Solar System and Beyond", 5th edn. Thomson Books Cole. 423 p. Slipher, V. (1914). Lowel Obs. Bull. 62, Vol. 11, 12. Cited in Sofu, Y. and Rubin V. (2001).

William, H.; Beyyer, (1985). "Standard Mathematical Tables". 7th edn., CRC Press Inc. pp. 253-254.

Wright, M.C.H. (1971). On the interpretation of observations of neural hydrogen in external galaxies. Ap. J. 166. 455 